\title{
Refractive state of eyes in dogs"1)
}

\section{AGNIESZKA BALICKA, IRENEUSZ BALICKI*, ALEXANDRA TRBOLOVA, MATEUSZ SZADKOWSKI*, ALEKSANDRA TOMKOWICZ**}

\author{
Small Animals Clinic, University of Veterinary Medicine and Pharmacy in Košice, \\ Komenského 7304181 Košice, Slovakia \\ *Department and Clinic of Animal Surgery, Faculty of Veterinary Medicine, \\ University of Life Sciences in Lublin, Akademicka 13, 20-950 Lublin, Poland \\ **Division of Small Animal Surgery and Anesthesiology, Faculty of Veterinary Medicine, \\ Warsaw University of Life Sciences, Nowoursynowska 159, 02-776 Warsaw, Poland
}

\section{Balicka A., Balicki I., Trbolova A., Szadkowski M., Tomkowicz A. Refractive state of eyes in dogs}

\section{Summary}

The article discusses refractive errors in dogs. The refractive errors presented in the article are myopia, hyperopia, anisometropia and astygmatism. These disadvantages are discussed in individual dogs breeds. Analysis has proved that most of dogs are emmetropic an thus have normal visual acuity. In dogs and human alike the refraction defect may have a genetic compound, it may be congenital or acquired and may change with age. Ametropia directly affects the orientation of animals and their behavior, therefore examination for refractive error is an important element of vision testing.

Keywords: myopia, hyperopia, anisometropia

Ocular refraction is the ability to refract light as it passes through the structures of the eye's optic system. In an emmetropic eye, the image passing through the optic system - composed of the cornea, aqueous humour, crystalline lens and vitreous body - is focused on the retina. In eyes with refractive disorders ametropia occurs. In such cases the eye cannot focus on the image on the retina. The image focused in front of or behind the retina results in blurred vision.

Refractive disorders include near-sightedness (myopia) and far-sightedness (hyperopia). Anisometropia is the condition where the refractive power for both two eyes is unequal $(2,6)$. The exact difference in dioptre has not been conclusively agreed on in the context of veterinary medicine. Currently, anisometropia is diagnosed for a $0.5 \mathrm{D}$ or $1.0 \mathrm{D}$ difference $(10$, 13). Modern ophtalmological diagnostics provides a number of methods for the examination of refractive disorders, including the once very common sciascopy, as well as the now increasingly popular autorefractometry. In the context of veterinary ophthalmology, a number of studies concerning refractive disorders have been documented, including studies conducted on small animals such as cats and dogs, as well as larger species, e.g. horses. Studies have also been carried out on rabbits, eagles, chickens, Guinea pigs and rodents: mice, rats.

\footnotetext{
1) The study was supported by the project VEGA 1/0225/15
}

A majority of dogs are emmetropic. Deviations from emmetropia may be related to an abnormal refractive strength of the crystalline lens, abnormal curvature of the cornea, or the length of the optical axis of the eye. The refraction defect observed in patients suffering from aphakia - i.e. absence of the crystalline lens - is $+14.4+/-2.1 \mathrm{D}$, which means that a dog can see an object located very far away as well as blurred images of objects located closer (6). Lens implants inserted into canine lens capsules during phacoemulsification surgeries have a strength of approximately $+41.5 \mathrm{D}(6$, $12,16,17)$. At the early beginning of the analysis it has been proved that only a lens of the power $+40.5-41.5$ (the difference of 1.5D is dependent on various breeds) corrects the refractive error in aphakic dogs. An intraocular lens that is placed in a capsular bag surrounded by an aqueous humor has a lower refractive power because of the small differences in refractive indices. That explains the cause why an aphakic patient with a refractive error of $+14.4 \mathrm{D}$ needs $+40.5 \mathrm{D}$ intraocular lens (17).

Objective analysis of refraction requires an individual approach to each animal species. It has been proved that refraction differences can be dependent on the breed and age of a particular dog, as well as the shape of the skull (17). Researchers from the University of Wisconsin, in collaboration with the College of Optometry, Ohio State University, analysed 
refractive disorders in 1,440 dogs of 90 breeds. Their studies revealed that refraction disorders are breed and age specific. The averaged result of the studies, generalised for all dogs regardless of the sex, breed or age, was indicative of emmetropia. It was demonstrated that myopia is most commonly observed in Rottweilers, Collies, miniature Schnauzers, and Toy Poodles. Meanwhile, hyperopia is most commonly diagnosed in Australian Shepherds as well as Bouvier des Flandres and Alaskan Malamute dogs. The authors concluded that although anisometropia affects only $6 \%$ of the canine population, in German Shepherds the defect is observed significantly more commonly: in $8.9 \%$ of the animals. Similarly to anisometropia, astigmatism was more common in German Shepherds $(3.3 \%)$ than in adult dogs of other breeds in the study $(1.0 \%)(10)$. In dogs and humans alike, regardless of gender, myopia can be exacerbated with age $(5,11$, $20)$. It was also demonstrated that in the population of guide dogs refractive disorders are far less common, which is most likely related to the many generations of selective breeding. The authors did note, however, that the criteria for this strict selection did not necessarily consider refractive disorders as such, but rather qualities such as general health or orientation, and therefore indirectly also refraction (10). Kennelled German Shepherds are a good example of the above as the incidence of myopia in kennelled dogs was shown to be smaller than in the case of non-kennelled German Shepherds (14).

Refractive disorders up to $-1.0 \mathrm{D}$ are not observable by the owners as sever visual acuity disorders. Visual acuity is not requiread and verified in dog's everyday activity - the dog will have no problems with negotiating obstacles, finding doors or locating its bowl (10). In the case of sporting dogs diagnosed with refractive disorders, it was observed that after applying corrective lenses (Proclear, Cooper Vision, Fairport, NY), the dogs had considerably fewer problems with spatial orientation during field trials (19). Moreover, it was noted that young dogs suffering from visual impairment require longer training when compared to emmetropic pups (10). It should also be pointed out that certain chemical compounds used in medicine may also cause visual acuity problems, including refractive disorders. For instance, dimethyl sulfoxide (DMSO) administered orally over extended periods of time caused crystalline lens opacification in dogs, which directly contributed to a change of the refractive index, leading to myopia. Similar problems have been reported in a study on dogs receiving oral doses of organic phosphorous pesticide (ethylthiometon) (10).

\section{Visual impairment in dogs of various breeds}

Toy Poodle, Springer Spaniel, Collies. Given the probable similarity between the pathomechanisms related to refraction disorders in humans and animals, researchers are particularly interested in analysing ame- tropia. The analysis of refractive disorders in animals may contribute to the understanding of the analogous problem in humans (4). Williams, Kubai, Murphy, and Mutti analysed ocular components in dogs with a high prevelence of myopia. The authors' research involving comparative studies of axial lengths and vitreous chamber depths revealed no significant differences between myopic and emmetropic dogs. In dogs suffering from myopia, the anterior crystalline lens radius was steeper $6.11+/-0.11 \mathrm{~mm}$, which resulted in a greater lens power $58.2+/-1.29 \mathrm{D})$. By comparison, the anterior crystalline lens radius in emmetropic dogs was 6.34 $+/-0.065 \mathrm{~mm}$ with a lens power of $55.5+/-0.77 \mathrm{D}$. Near-sighted Springer Spaniels were an exception in this respect, with the reported greater mean lens thickness when compared to non-myopic dogs of the same breed. Additionally, it was observed that the anterior chamber depth in myopic dogs $(3.28+/-0.84 \mathrm{~mm})$ was similar to that observed in pups $(3.20+/-0.75 \mathrm{~mm})$. In emmetropic dogs, the anterior chamber was observed to deepen with age: it was $3.21+/-0.66 \mathrm{~mm}$ in young dogs and $3.88+/-0.74 \mathrm{~mm}$ in adult animals. Authors studying myopia in Toy Poodles, English Springer Spaniels and Collie dogs suggest that near-sightedness is lenticular in origin, unlike in the case of Labrador Retrievers where it correlates with the length of the vitreous chamber, a fact of considerable significance for researchers working on inhibiting the development of myopia in humans (24).

German Shepherds. German Shepherds are known to be susceptible to myopia. The defect has been reported in $53 \%$ of non-kennelled dogs. The defect severity of $-0.86+/-1.31 \mathrm{D}$ is significantly higher in comparison to working dogs in which the reported value is $+0.22+/-0.79$, with only $19 \%$ of the animals suffering from myopia (14). A study involving corneal curvature measurements was conducted on German Shepherds but no significant differences between emmetropic and ametropic dogs were observed. It is believed that crystalline lens changes are the main cause of refraction disorders in German Shepherds. As to comparative analyses of eyesight test results and anatomical measurements, the axial lengths in emmetropic and ametropic animals of this breed revealed no significant differences (14).

Beagles. In 2011, a study was conducted to determine what refractive disorders occur in Beagles. Even though EU legislation allows experiments on Beagles, no studies on the incidence of refraction disorders in this breed of dogs had previously been conducted. The mean result reported from the study conducted with the use of sciascopy was indicative of emmetropia, i.e. a majority of dogs of this breed do not suffer from eyesight problems related to refractive disorders. It was also noted, however, that where myopia was observed in Beagles, the condition grew more severe with age. In animals aged 8-9 years, compared to 3-6 year old Beagles, myopia was significantly more advanced. 
Similar results have also been reported from studies on other breeds, which may be interpreted as an agerelated tendency in dogs. It is suspected that myopia in Beagles may be related to sclerosis of the crystalline lens nucleus that changes its focusing power (13).

Labrador Retriever. Myopia models in humans and Labrador Retrievers have been shown to be analogous and directly related to increased length of the vitreous chamber. However, no excessive overall axial elongation of the eye was reported in humans suffering from myopia. Researchers also reported a thinning of the crystalline lens in myopic Labrador Retrievers, which corresponded to similar thinning observed in humans. In near-sighted Labrador Retrievers measurements indicated that the lens was more asymmetric, with the anterior surface being steeper than the posterior surface (15).

Although most Labrador Retrievers are emmetropic, myopia is diagnosed in 8-15\% of animals of this breed, which is a relatively high index when compared to other breeds $(2,15)$. A study on 75 Labrador Retrievers was conducted with the aim of analysing the inheritance of myopic traits naturally occurring within this breed. In 11 dogs $(14.7 \%)$ myopia was diagnosed in at least one eye, and in $6(8 \%)$ in both eyes. The severity of refractive disorders diagnosed in the dogs was between +3.5 and $-5.0 \mathrm{D}$. The results confirmed that myopia in Labrador Retrievers is related to the elongation of the vitreous chamber and a thinning of the lens, rather than to the focusing power of the lens (15). Not advanced myopia occurs naturally in dogs. It is hereditary and relatively uncommon, although exactly how uncommon depends on the particular breed. Studies on Labrador Retrievers indicated that the incidence of myopia may be conditioned genetically but is also related to such factors as the number of pups in a given litter as well as environmental factors which are yet to be identified in the course of further research (2). It seems that in dogs from smaller litters myopia occurs more commonly than in animals born in larger litters. Evidence in humans suggests that increased activity tends to delay the onset of myopia $(9,21)$. It is suspected that a similar mechanism comes into play in newly born pups which are forced to engage in more intensive activity when born to larger litters (2). Researchers are particularly keen to study myopia in Labrador Retrievers as its pathomechanism is largely similar to that observed in humans and involves the elongation of the vitreous chamber. Studies conducted on consecutive generations of Labrador Retrievers are intended to further our understanding of hereditary myopia in humans, as well as to identify the so-called risk factors (2). To date, it has been determined that myopia in humans can also result from a number of factors. Environmental risk factors include: work in rooms illuminated with artificial light, looking at objects from very small distances over extended periods of time. Near-sightedness can also be genetically conditioned.
It is commonly recognised that children of myopic parents are significantly more likely to wear corrective glasses than children of emmetropic parents $(1,3)$.

Rottweiler and Schnauzer. Studies conducted on consecutive generations of miniature Schnauzer and Rottweiler dogs were aimed at determining the incidence of refractive disorders among dogs from these breeds. It was shown that there are generations of dogs where myopic animals inherited the trait from an ancestor suffering from the same problem. Analogous research conducted simultaneously on miniature Schnauzers and Rottweilers without refractive disorders indicated that the subsequent generations also did not display a myopic tendency. The research confirms earlier suspicions that at least in some dog breeds myopia is indeed hereditary.

As many as $64 \%$ of the studied Rottweilers were diagnosed with moderate myopia with the mean severity of $-1.77+/-1.84 \mathrm{D}$, with the average value of refractive disorders in all near-sighted animals of this breed calculated at $-2.89+/-1.27 \mathrm{D}$. In Schnauzers, myopia was diagnosed in in half of the population. The mean value obtained from refraction examinations was -0.67 $+/-1.05 \mathrm{D}$, and in myopic animals $-1.50+/-0.65 \mathrm{D}(14)$.

\section{Treatment of retinal detachment and refractive disorders}

In dogs suffering from retinal detachment, the recommended course of treatment is to apply silicone oil ( $\mathrm{SiO})$ into the vitreous chamber. Despite many possible complications, including displacement of the anterior chamber, keratopathy and glaucoma, the method is widely recommended due to its potential to significantly improve the patient's quality of life. Application of silicone oil is not a method commonly available in veterinary medicine.

In all dogs that underwent silicone oil tamponade as a result of complete retinal detachment, hyperopia was observed regardless on the actual refractive power of the lens. In treated patients the severity of refractive disorders in phakic animals averaged at $+2.67 \mathrm{D}$, in pseudophakic animals $+3.24 \mathrm{D}$, and $+6.5 \mathrm{D}$ in aphakic animals. The refraction disorder stems from the higher refraction index of $\mathrm{SiO}$ when compared to the vitreous body. This particular disadvantage can be corrected by applying silicone oils of different viscosity, depending on the given patient's needs (8). It has also been reported in medicine that the degree of hyperopia changes relative to the position of the head. When leaning over (with the face toward the ground) the curvature of the anterior $\mathrm{SiO}$ surface is reduced (7). The authors recommend the use of $\mathrm{SiO}$ despite the mentioned complications and emphasise the vital importance of good communication with the patient's owner to explain all the relevant aspects of the treatment, including anisometropia. Silicone oil is not usually removed after application in dogs, as the procedure runs the risk of retinal re-detachment (8). In 
humans, the oil is removed 2-6 months after the procedure to minimise the risk of cataract or keratopathy and to correct anisometropia $(20,23)$. Anisometropia occurs when the refraction defect in the eye treated with $\mathrm{SiO}$ is different from that in the other, potentially healthy eye (8).

\section{Influence of refractive error on dog's orientation}

A study conducted in 2012 provided better understanding of how problems related to refraction can directly influence dogs' spatial awareness. During field trials, the results of 8 dogs were recorded and analysed (7 Labrador Retrievers and 1 Chesapeake Bay Retriever). The analysis was performed on the basis of the objective and subjective results achieved by the particular dogs. The objective result was the time needed to complete the course. The subjective result was the score given by 2 experienced judges. Each dog underwent three trials. Before each trial contact lenses were put in both eyes of each dog, respectively $0 \mathrm{D},+1.5 \mathrm{D}$ and +3.0D. At the end of each run it was verified that the dog did not loose a contact lens in the process. Six of the original 14 dogs had to be excluded from the study for this reason. During the search trial, dogs wearing $0 \mathrm{D}$ lenses needed no help whatsoever from their owners. With $+1.5 \mathrm{D}$ and $+3 \mathrm{D}$ lenses respectively, 2 and 3 dogs needed assistance before they could localise the target. It was also observed that the dogs needed more time to traverse the course when wearing $+1.5 \mathrm{D}$ and $+3 \mathrm{D}$ lenses (there were no significant differences between timing rankings for when +1.50 - and +3.00 -D lenses were worn). The judges' observations also indicated that dogs wearing the most powerful lenses $(+3.0 \mathrm{D})$ to imitate a refraction defect displayed worse orientation and received lower scores when compared to $+1.5 \mathrm{D}$ lenses, although $+1.5 \mathrm{D}$ lenses also hindered orientation when compared to the 0D lenses, which caused no problems. The study demonstrated that refractive disorders have a significant impact on dogs' orientation and behaviour in their environment. The authors suggest that refraction examinations ought to be taken into consideration in the process of selecting future working dogs, e.g. guide dogs trained to assist the blind.

Research on refractive disorders in animals is becoming increasingly prevalent in veterinary medicine. One of the goals in contemporary research is to identify methods of inhibiting and preventing the development of myopia in humans (19). A great number of people all around the world, including children and the elderly, are suffering from near-sightedness. In extreme cases, consequences attributable to myopia can include retinal detachment, glaucoma or cataract $(11,22)$. Finding an animal model of myopia that would closely resemble that observed in humans would help researchers to gain a deeper insight into various aspects of this problem. The diagnosis of particular refractive disorders in animals allows their owners to better understand certain behaviours of their dogs, cats or horses and limit certain expectations they may have towards their animals.

It is the task of the veterinarian to inform the patient's owner of the specificity of refractive disorders and their effects on animals. It is important that the owner understands these problems, particularly since the animal's spatial orientation is not based exclusively on its eyesight, and its broadly understood ability to see is to a large extent different from that experienced by humans. The prevalence of rod cells over cone cells provides many mammals with enhanced night vision. It must be remembered, however, that the resulting reduction in the number of cone cells negatively affects visual acuity (18). To improve the quality of patients' lives, lenses can be implanted into the lens capsule during phacoemulsification surgery with the aim of preventing refractive disorders after removing the masses of cataract lens. Corrective lenses for dogs are available on the market of veterinary accessories. These are available in a variety of sizes depending on the size of the dog, and are intended for use in animals suffering from aphakia. Bandage lenses are currently also more commonly used in veterinary medicine. Although they do not correct refractive disorders, they are recommended for patients with corneal ulcerations or in cases of pre- or post-operative care. Lenses in various sizes are also available on special order for dogs, cats and horses. A lens ruler can be helpful in selecting the correct lens by determining the appropriate lens size. UV filter lenses and sunglasses are also available for patients suffering, for example, from chronic superficial keratitis. This type of UV filter protective glasses are commonly used by police or military dogs during fieldwork.

To date, studies on refractive disorders in animals are few and far between despite the fact that a diagnosis of a particular refraction disorder provides information relevant to the owner's realistic expectations towards an animal, both in terms of work and play.

\section{References}

1. Biino G., Palmas M. A., Corona C., Prodi D., Fanciulli M., Sulis R., Serra A., Fossarello M., Pirastu M.: Ocular refraction: heritability and genome-wide search for eye morphometry traits in an isolated Sardinian population. Hum. Genet. 2005, 116,152-159.

2. Black J., Browning S. R., Collins A. V., Phillips J. R.: Canine Model of Inherited Myopia: Familial Aggregation of Refractive Error in Labrador Retrievers. Invest. Ophthalmol. Vis. Sci. 2008, 49, 4784-4789.

3. Chen C. Y., Scurrah K. J., Stankovich J., Garoufalis P., Dirani M., Pertile K. K., Richardson A. J., Mitchell P., Baird P. N.: Heritability and shared environment estimates for myopia and associated ocular biometric traits: the Genes in Myopia (GEM) family study. Hum. Genet. 2007, 121, 511-520.

4. Coile D. C., O'Keefe L. P.: Schematic eyes for domestic animals. Ophthalmic Physiol. Opt. 1988, 8, 215-220.

5. Committee on Vision of the National Research Council Commission on Behavioral and Social Sciences and Education: Working group report on myopia prevalence and progression. National Academy Press, Washington 1989.

6. Davidson M. G., Murphy C. J., Nasisse M. P., Hellkamp A. S., Olivero D. K., Brinkmann M. C., Campbell L. H.: Refractive state of aphakic and pseudophakic eyes of dogs. Am. J. Vet. Res. 1993, 54, 174-177.

7. Dick H. B., Schwenn O., Pavlovic S., Jacobi F. K., Pfeiffer N.: Effect of head position on refraction in aphakic and phakic silicone-filled eyes. Retina. 1997, $17,397-402$ 
8. Hoffman A., Wolfer J., Occelli L., Lehenbauer T. W., Sapienza J., NovakJ. M., Combs K. L., Konrade K. A.: Refractive state following retinal reattachment and silicone oil tamponade in dogs. Am. J. Vet. Res. 2012, 73, 1299-1304.

9. Jacobsen N., Jensen H., Goldschmidt E.: Does the level of physical activity in university students influence development and progression of myopia? a 2-year prospective cohort study. Invest. Ophthalmol. Vis. Sci. 2008, 49, 1322-1327.

10. Kubai M. A., Bentley E., Miller P. E., Mutti D. O., Murphy C. J.: Refractive states of eyes and association between ametropia and breed in dogs. Am. J. Vet. Res. 2008, 69, 946-951.

11. Lim R., Mitchell P., Cumming R. G.: Refractive associations with cataract: the Blue Mountains Eye Study. Invest. Ophthalmol. Vis. Sci. 1999, 40, 3021-3026.

12. Lowe R.: The lens, Gould D., McLellan G. J.: BSAVA Mannual of Caninie and Feline Ophthalmology. BSAVA, Gloucester 2015, p. 297-313.

13. Maehara S., Itoh Y., Higashinozono K., Izumisawa Y.: Evaluation of refractive value by skiascopy in healthy Beagle dogs. J. Vet. Med. 2011, 73, 927-929.

14. Murphy C. J., Zodnik K., Mannisf M. J.: Myopia and Refractive Error in Dogs. Invest. Ophthalmol. Vis. Sci. 1992, 33, 2459-2463.

15. Mutti D., Zodnik K., Murphy C. J.: Naturally Occurring Vitreous ChamberBased Myopia in the Labrador Retriever. Invest. Ophthalmol. Vis. Sci. 1999, 40, 1577-1584.

16. Ofri R.: Lens [in:] Maggs D. J., Miller P. E., Ofri R.: Slatter's Fundamentals of Veterinary Ophthalmology. Elsevier, St. Louis 2013, p. 272-290.
17. Ofri R.: Optics and Physiology of Vision, [in:] Gelatt K. N., Gilger B. C., Kern T. J.: Veterinary Ophthalmology. Wiley-Blackwell, New Jersey 2013, p. 183-219.

18. Ofri R.: Retina [in:] Maggs D. J., Miller P. E., Ofri R.: Slatter's Fundamentals of Veterinary Ophthalmology. Elsevier, St. Louis 2013, p. 299-333.

19. Ofri R., Hollingsworth S. R., Groth A., Motta M. J., Doval J. H., Kass P. H., Murphy C. J.: Effect of optical defocus on performance of dogs involved in field trial competition. Am. J. Vet. Res. 2012, 73, 546-550.

20. Panchapakesan J., Rochtchina E., Mitchell P.: Myopic refractive shift caused by incident cataract: the Blue Mountains Eye Study. Ophthalmic Epidemiol. 2003, 10, 241-247.

21. Rose K. A., Morgan I. G., Ip J., Kifley A., Huynh S., Smith W., Mitchell P.: Outdoor activity reduces the prevalence of myopia in children. Ophthalmology 2008, 115, 1279-1285.

22. The Eye Disease Case-Control Study Group. Risk factors for idio-pathic rhegmatogenous retinal detachment. Am. J. Epidemiol. 1993, 137, 749-757.

23. Valone J., McCarthy M.: Emulsified anterior chamber silicone oil and glaucoma Ophthalmology 1994, 101, 1908-1912.

24. Williams L. A., Kubai M. A., Murphy C. J., Mutti D. O.: Ocular Components in Three Breeds of Dogs with High Prevalence of Myopia. Optometry \& Vision Science 2011, 88, 269-274.

Corresponding author: Agnieszka Balicka, DVM: e-mail: agnieszka.a. balicka@gmail.com 